\title{
Adaptive Control using Reduced-Order Observers
}

\author{
Jovan D. Bošković and Nathan Knoebel \\ Scientific Systems Company, Inc., 500 West Cummings Park, Suite 3000, Woburn, MA 01801 \\ Email: jovanessci.com, nknoebelessci.com
}

\begin{abstract}
In many practical situations uncertain plants are such that the unknown parameters do not affect the entire state of the system, but only some of the state variables. A question that arises in this context is the following: can a reduced-order adaptive observer be designed based only on the part of the dynamics affected by the uncertainty, such that, when the corresponding parameter estimates are used in the control law, the closed-loop stability is guaranteed? The related objective is to design the adaptive observer that has the number of adjustable parameters equal to the number of uncertain paramaters, and whose order coincides with the lowest-order subsystem affected by the uncertainty.

In this paper a new systematic procedure is developed for the design of stable adaptive controllers using local reducedorder adaptive observers. It is shown that, for the class of plants considered in the paper, even when the unknown parameters are estimated using such lower-order observers, the resulting closed-loop system will be stable, and the asymptotic convergence of the tracking error to zero is guaranteed.
\end{abstract}

\section{INTRODUCTION}

As it is well known, most of the adaptive control schemes can be classified into direct and indirect [1]. In direct adaptive control controller gain matrices are adjusted directly based on the response of the closed-loop system, and the controller parameter adjustment based on Lyapunov analysis results in a stable system in which the convergence of the tracking error to zero is guaranteed. One of the features of this approach is that the Lyapunov matrix equation needs to be solved (offline) to calculate a matrix $P$ that is needed to implement the adaptive laws. In indirect adaptive control a suitable observer is built and used to adjust the estimates of unknown parameters. These estimates are in turn used in the control law to assure the boundedness of all the signals in the system and asymptotic convergence of the estimation and tracking errors to zero.

In the present literature it is commonly assumed that the adaptive observer is of the same order as the uncertain plant. However, in many practical situations the dynamics of only a part of the system state is affected by the uncertain parameters. A good example is a system in the controllable canonical form where only relative degree one states are affected by uncertain paramaters. A question that arises in this context is whether it is possible to design a stable adaptive scheme using a reduced order observer for unknown parameters. The design of such an observer is the main focus of this paper.

\section{AdAPtive Control USing Reduced-ORder OBSERVERS}

In many practical situations the uncertain parameters do not affect the entire state of the system, but only some of the state variables. One good exaple is the system in the controllable canonical form with the so-called matched uncertainties (i.e. the uncertainties that appear in the same equations as the control inputs). In that case the states with the relative-degree two and higher are not affected by the uncertainties. In this paper the main objective is to demonstrate that it is possible to design the observer that has the number of adjustable parameters equal to the number of uncertain paramaters, and whose order coincides with the lowest-order subsystem affected by the uncertainty.

In order to demonstrate the main ideas, the problem will be divided into two parts: (i) Relative degree one plants; and (ii) Plants with relative degree two.

\section{A. Relative Degree One Plants}

In this case the plant dynamics is described by:

$$
\dot{x}=\left[\begin{array}{ll}
\bar{A}_{11} & \bar{A}_{12} \\
A_{21} & A_{22}
\end{array}\right] x+\left[\begin{array}{c}
\bar{B}_{1} \\
B_{2} D
\end{array}\right] u
$$

where $x: \mathbb{R}^{+} \rightarrow \mathbb{R}^{n}, x=\left[\begin{array}{ll}x_{1}^{T} & x_{2}^{T}\end{array}\right]^{T}, x_{1}: \mathbb{R}^{+} \rightarrow \mathbb{R}^{n_{1}}$, $x_{2}: \mathbb{R}^{+} \rightarrow \mathbb{R}^{n_{2}}, n_{1}+n_{2}=n, u: \mathbb{R}^{+} \rightarrow \mathbb{R}^{m}, \bar{A}_{11}, A_{21} \in$ $\mathbb{R}^{n_{1} \times n_{1}}, \bar{A}_{12}, A_{22} \in \mathbb{R}^{n_{2} \times n_{2}}, B_{1} \in \mathbb{R}^{n_{1} \times m}, B_{2} \in \mathbb{R}^{n_{2} \times m}$, and $D=\operatorname{diag}(d)$ where $d=\left[\begin{array}{llll}d_{1} & d_{2} & \ldots & d_{m}\end{array}\right]^{T}$. It is assumed that $A_{21}, A_{22}$ and $D$ are unknown, while all other matrices are known accurately.

\section{Assumption 1:}

(i) State of the system is measurable.

(ii) $d \in \mathcal{S}_{d}=\left\{d: 0<\epsilon_{i} \leq d_{i} \leq 1, i=1,2, \ldots, m\right\}$.

(iii) Let $\tilde{B}=\left[B_{1}^{T}\left(B_{2} D\right)^{T}\right]^{T}$. Then $\operatorname{rank}(\tilde{B})=n$ for all $d \in \mathcal{S}_{d}$.

The objectives are:

1. Design a control signal $u(t)$ such that the state of the plant follows asymptotically that of the following reference model:

$$
\dot{x}_{m}=\left[\begin{array}{cc}
A_{11}^{*} & A_{12}^{*} \\
A_{21}^{*} & A_{22}^{*}
\end{array}\right] x_{m}+\left[\begin{array}{c}
B_{1}^{*} \\
B_{2}^{*}
\end{array}\right] r
$$

where $x_{m}: \mathbb{R}^{+} \rightarrow \mathbb{R}^{n}, x_{m}=\left[x_{m 1}^{T} x_{m 2}^{T}\right]^{T}, x_{m 1}: \mathbb{R}^{+} \rightarrow$ $\mathbb{R}^{n_{1}}, x_{m 2}: \mathbb{R}^{+} \rightarrow \mathbb{R}^{n_{2}}, n_{1}+n_{2}=n$, denote the state of the reference model, $r: \mathbb{R}^{+} \rightarrow \mathbb{R}^{n}$ is a vector of bounded 
piecewise continuous reference inputs, the matrix

$$
A_{m}=\left[\begin{array}{cc}
A_{11}^{*} & A_{12}^{*} \\
A_{21}^{*} & A_{22}^{*}
\end{array}\right],
$$

is asymptotically stable, and $\operatorname{rank}\left(B_{m}\right)=n$.

2 . Use only the $x_{2}$ equation to design an adaptive observer as a part of the overall adaptive control scheme.

Ideal Controller: Let:

$A=\left[\begin{array}{cc}\bar{A}_{11} & \bar{A}_{12} \\ A_{21} & A_{22}\end{array}\right], B=\left[\begin{array}{c}B_{1} \\ B_{2} D\end{array}\right], B_{m}=\left[\begin{array}{c}B_{1}^{*} \\ B_{2}^{*}\end{array}\right]$.

In the case when $A_{21}, A_{22}$ and $D$ are known, the following controller achieves the objective:

$$
u=B^{T}\left(B B^{T}\right)^{-1}\left[-A x+A_{m} x+B_{m} r\right] .
$$

\section{B. Parameter Estimation}

In the case when these matrices are unknown, their estimation will be carried out using the following reduced-order adaptive observer:

$$
\dot{\hat{x}}_{2}=\hat{A}_{2} x+B_{2} U \hat{d}+\Lambda \hat{e}_{2},
$$

where $\hat{e}_{2}=\hat{x}_{2}-x_{2}, U \hat{d}=\hat{D} u, A_{2}=\left[\begin{array}{ll}A_{21} & A_{22}\end{array}\right]$, and $\Lambda=\Lambda^{T}<0$.

Let $\Phi_{A}=\hat{A}_{2}-A_{2}$ and $\phi_{d}=\hat{d}-d$. It can now be shown using standard arguments [1] that the adaptive algorithms:

$$
\begin{aligned}
\dot{\hat{A}}_{2}=\dot{\Phi}_{A} & =-\Gamma_{A} \hat{e}_{2} x^{T}, \\
\dot{\hat{d}}=\dot{\phi}_{d} & =\operatorname{Proj}_{\mathcal{S}_{d}}\left\{-\Gamma_{d} B_{2} U \hat{e}_{2}\right\},
\end{aligned}
$$

where $\Gamma_{A}=\Gamma_{A}^{T}>0$ and $\Gamma_{d}=\Gamma_{d}^{T}>0$, result in $\hat{A}_{2}, \hat{d} \in \mathcal{L}^{\infty}$ and $\hat{e}_{2} \in \mathcal{L}^{\infty} \cap \mathcal{L}^{2}$. The objective now is to show that the use of the parameter estimates, generated by the reduced-order observer, in the control law will result in a stable system and that the control objective will be met. This is discussed in the following section.

\section{Adaptive Control}

Let:

$$
\hat{A}=\left[\begin{array}{cc}
\bar{A}_{11} & \bar{A}_{12} \\
\hat{A}_{21} & \hat{A}_{22}
\end{array}\right], \hat{B}=\left[\begin{array}{c}
B_{1} \\
B_{2} \hat{D}
\end{array}\right]
$$

and let the corresponding certainty-equivalence adaptive controller be of the form:

$$
u=\hat{B}^{T}\left(\hat{B} \hat{B}^{T}\right)^{-1}\left[-\hat{A} x+A_{m} x+B_{m} r\right] .
$$

Now the following theorem is considered:

Theorem II.1: Closed-loop system (1), (6), (3), (4), (5) is stable, and $\lim _{t \rightarrow \infty} e(t)=0$.

Proof: The following coordinate transformation is chosen:

$$
z_{1}=x_{1}, \quad z_{2}=\hat{x}_{2},
$$

so that $z=\left[\begin{array}{ll}z_{1}^{T} & z_{2}^{T}\end{array}\right]^{T}=\left[\begin{array}{ll}x_{1}^{T} & \hat{x}_{2}^{T}\end{array}\right]^{T}$.

It follows that:

$$
\begin{aligned}
& \dot{z}_{1}=\bar{A}_{11} x_{1}+\bar{A}_{12} x_{2}+B_{1} u \\
& \dot{z}_{2}=\hat{A}_{21} x_{1}+\hat{A}_{22} x_{2}+B_{2} \hat{D} u+\Lambda_{2} \hat{e}_{2} .
\end{aligned}
$$

After substituting the control law (6) one obtains:

$$
\dot{z}=A_{m} z+B_{m} r+M \hat{e}_{2},
$$

where $M=\left[\begin{array}{ll}-A_{12}^{* T} & \Lambda_{2}-A_{22}^{* T}\end{array}\right]^{T}$. Upon defining $e_{m}=$ $z-x_{m}$ and subtracting the reference model equation, one obtains:

$$
\dot{e}_{m}=A_{m} e_{m}+M \hat{e}_{2} .
$$

It is noted that $e_{m 1}=e_{1}$. Since $\hat{e}$ is bounded, it follows that $e_{m}=z-x_{m}$ and $z$ are bounded, as well as $\hat{x}$. It can now be readily shown that all the signals in the system are bounded and that $\lim _{t \rightarrow \infty} e(t)=0$.

The main feature of the proposed adaptive control scheme is that the number of parameters that needs to be adjusted is equal to the number of unknown parameters. The order of the associated adaptive observer is equal to the order of the largest subsystem affected by the uncertainty. This results in substantially decreased computation if a small number of parameters are unknown.

Comment: The proposed approach for relative degree one plants can be readily extended to the controllable canonical form with matched uncertainties:

$$
\begin{aligned}
\dot{x}_{1} & =x_{2} \\
\dot{x}_{2} & =x_{3} \\
& \vdots \\
\dot{x}_{n-1} & =x_{n} \\
\dot{x}_{n} & =A x+B D u,
\end{aligned}
$$

where $A$ and $D$ are unknown and $\operatorname{rank}(B D)=\operatorname{dim}\left(x_{n}\right)$. The observer is based on the last equation only:

$$
\dot{\hat{x}}_{n}=A x+B U \hat{d}+\Lambda \hat{e}_{n}
$$

where $\Lambda=\Lambda^{T}<0$. Adaptive laws are of the form:

$$
\begin{aligned}
\dot{\hat{A}} & =-\Gamma_{A} \hat{e}_{n} x^{T} \\
\dot{\hat{d}} & =\operatorname{Proj}_{\mathcal{S}_{d}}\left\{-\Gamma_{d} U B^{T} \hat{e}_{n}\right\},
\end{aligned}
$$

and the stability can be proved using the following coordinate transformation:

$$
z_{1}=\bar{x}, \quad z_{2}=\hat{x}_{n},
$$

where $\bar{x}=\left[\begin{array}{llll}x_{1}^{T} & x_{2}^{T} & \ldots & x_{n-1}^{T}\end{array}\right]^{T}$. The state of the system will follow the following reference model:

$$
\begin{aligned}
\dot{x}_{1}^{*} & =x_{2}^{*} \\
\dot{x}_{2}^{*} & =x_{3}^{*} \\
& \vdots \\
\dot{x}_{n-1}^{*} & =x_{n}^{*} \\
\dot{x}_{n}^{*} & =-\sum_{i=1}^{n} k_{i} x_{i}^{*}+k_{1} r,
\end{aligned}
$$

where $k_{i}$ are chosen to assure the stability of the reference model. 


\section{Plants of Relative Degree Two with UNMATCHED UNCERTAINTIES}

In this case the plant dynamics is of the form:

$$
\dot{x}=\left[\begin{array}{ll}
A_{11} & A_{12} \\
A_{21} & A_{22}
\end{array}\right] x+\left[\begin{array}{c}
0 \\
B_{2}
\end{array}\right] u,
$$

where $x: \mathbb{R}^{+} \rightarrow \mathbb{R}^{n}, x=\left[\begin{array}{ll}x_{1}^{T} & x_{2}^{T}\end{array}\right]^{T}, x_{1}: \mathbb{R}^{+} \rightarrow \mathbb{R}^{n_{1}}$, $x_{2}: \mathbb{R}^{+} \rightarrow \mathbb{R}^{n_{2}}, n_{1}+n_{2}=n, u: \mathbb{R}^{+} \rightarrow \mathbb{R}^{m}, A_{11}, A_{21} \in$ $\mathbb{R}^{n_{1} \times n_{1}}, A_{12}, A_{22} \in \mathbb{R}^{n_{2} \times n_{2}}$, and $B_{2} \in \mathbb{R}^{n_{2} \times m}$. It is assumed that only $A_{11}$ is unknown, while all other matrices are known accurately. Hence one of the objectives is to design an adaptive observer based on the $x_{1}$ equation only. Also, the extensions to the case when a part of the state $x_{2}$ also affected by uncertainty are straightforward. The above system corresponds to the systems in the so-called triangular form (see e.g. [3]), where a part of the uncertainty is not matched (i.e. it does not appear in the same equation as $u$ ), but is associated only with the variables of relative degree two or higher.

\section{Assumption 2:}

(i) State of the system is measurable.

(ii) $\operatorname{rank}\left(A_{12} B_{2}\right)=n_{1}$.

For the Assumption 2(ii) to hold, a necessary condition is that $n_{1}=n_{2}$. While this appears fairly restrictive, some important classes of plants satisfy this assumption, for instance:

$$
\begin{aligned}
& \dot{x}_{1}=x_{2}+A_{11} x_{1} \\
& \dot{x}_{2}=A_{21} x_{1}+A_{22} x_{2}+B_{2} u,
\end{aligned}
$$

which, for $A_{11}=0$, reduces to the controllable canonical form. In addition, most of the models describing the dominant dynamics of aircraft, spacecraft, helicopters and other systems are of multivariable relative degree two.

Control Objective: The objective is to design a control signal $u(t)$ such that the state $x_{2}(t)$ is bounded and the state $x_{1}(t)$ of the plant follows asymptotically the state $x_{m 1}(t)$ of the reference model:

$$
\begin{aligned}
& \dot{x}_{m 1}=x_{m 2} \\
& \dot{x}_{m 2}=-K_{1} x_{m 1}-K_{2} x_{m 2}+K_{1} r,
\end{aligned}
$$

where the matrices $K_{1}, K_{2}>0$ are chosen such that the matrix

$$
A_{m}=\left[\begin{array}{cc}
0 & I \\
-K_{1} & -K_{2}
\end{array}\right]
$$

is asymptotically stable.

The main difficulty in this case is the fact that $A_{11}$ is unknown, and appears in the equation not affected by $u$. In this case there are several possible approaches to the design of stable adaptive controllers. One of those is the so-called back-stepping [3] which results in a complex design, and a full-order observer is needed to estimate the parameters. The focus here will be on approaches that are based on reducedorder observers. Two possible approaches will be considered as discussed below - one without an explicit observer, and the other one with a reduced-order observer. Before that the ideal controller is derived first.

Ideal Controller: The following coordinate transformation is introduced:

$$
\begin{aligned}
z_{1}^{*} & =x_{1} \\
z_{2}^{*} & =A_{11} x_{1}+A_{12} x_{2} .
\end{aligned}
$$

Upon differentiating $x_{1}$ from (8), one obtains:

$\dot{z}_{1}^{*}=z_{2}^{*}$

$\dot{z}_{2}^{*}=\left(A_{11}^{2}+A_{12} A_{21}\right) x_{1}+\left(A_{11} A_{12}+A_{12} A_{22}\right) x_{2}+A_{12} B_{2} u$.

The ideal controller is now chosen as:

$$
\begin{aligned}
u= & \left(A_{12} B_{2}\right)\left(A_{12} B_{2}\left(A_{12} B_{2}\right)^{T}\right)^{-1}\left(-\left(A_{11}^{2}+A_{12} A_{21}\right) x_{1}\right. \\
& \left.-\left(A_{11} A_{12}+A_{12} A_{22}\right) x_{2}+A_{m} z^{*}+B_{m} r\right) .
\end{aligned}
$$

It is seen that $z^{*}$ rather than $x$ is used in the control law. This is because the entire state of the system cannot follow a reference model in a controllable form, and only a part of the system (i.e. $x_{1}$ ) can follow a part of the reference model state (i.e. $x_{m 1}$ ).

The resulting closed-loop system is now:

$$
\dot{z}^{*}=A_{m} z^{*}+B_{m} r
$$

so that $\lim _{t \rightarrow \infty}\left[z^{*}(t)-x_{m}(t)\right]=0$, which implies that $\lim _{t \rightarrow \infty}\left[x_{1}(t)-x_{m 1}(t)\right]=0$, while $x_{2}(t)$ is bounded.

Local Observer: In this case the observer is chosen in the form:

$$
\dot{\hat{x}}_{1}=\hat{A}_{11} x_{1}+A_{12} x_{2}+\Lambda_{1} \hat{e}_{1},
$$

where, to simplify the development, $\Lambda_{1}<0$ is chosen as a diagonal matrix, and $\hat{e}_{1}=\hat{x}_{1}-x_{1}$. Hence one can choose $\Lambda_{1}=-\Lambda$, where $\Lambda=\operatorname{diag}\left[\begin{array}{llll}\lambda_{1} & \lambda_{2} & \ldots & \lambda_{n_{1}}\end{array}\right]$ and $\lambda_{i}>0$.

Error Model: Upon subtracting (8) from the above expression, one obtains:

$$
\dot{\hat{e}}_{1}=-\Lambda \hat{e}_{1}+\Phi_{A} x_{1},
$$

where $\Phi_{A}=\hat{A}_{11}-A_{11}$.

The following coordinate transformation is now chosen:

$$
\begin{aligned}
& z_{1}=x_{1}, \\
& z_{2}=\hat{A}_{11} x_{1}+A_{12} x_{2},
\end{aligned}
$$

resulting in:

$$
\begin{aligned}
\dot{z}_{1}= & z_{2}-\Phi_{A} x_{1} \\
\dot{z}_{2}= & \dot{\hat{A}}_{11} x_{1}+\hat{A}_{11}\left[\left(\hat{A}_{11}-\Phi_{A}\right) x_{1}\right. \\
& \left.\quad+A_{12} x_{2}\right]+A_{12}\left(A_{21} x_{1}+A_{22} x_{2}+B_{2} D u\right) \\
= & \dot{\hat{A}}_{11} x_{1}+\hat{A}_{11}^{2} x_{1}+\hat{A}_{11} A_{12} x_{2}-\hat{A}_{11} \Phi_{A} x_{1} \\
& +A_{12}\left(A_{21} x_{1}+A_{22} x_{2}+B_{2} D u\right) .
\end{aligned}
$$

The control law is now chosen in the form:

$$
\begin{aligned}
u= & B^{T}\left(B B^{T}\right)^{-1}\left(-\dot{\hat{A}}_{11} x_{1}-\hat{A}_{11}^{2} x_{1}-\hat{A}_{11} A_{12} x_{2}\right. \\
& \left.-A_{12}\left(A_{21} x_{1}+A_{22} x_{2}\right)+v\right),
\end{aligned}
$$


where $v=-K_{1} z_{1}-K_{2} z_{2}+K_{1} r$. It is seen that the term $\dot{\hat{A}}_{11}$ is kept as is for the time being. Upon substituting this control law and subtracting the reference model, one obtains the following error model:

$$
\begin{aligned}
& \dot{e}_{1}=e_{2}-\Phi_{A} x_{1} \\
& \dot{e}_{2}=-K_{1} e_{1}-K_{2} e_{2}-\hat{A}_{11} \Phi_{A} x_{1},
\end{aligned}
$$

or

$$
\dot{e}=A_{m} e-\hat{B}_{0} \Phi_{A} x_{1} .
$$

Theorem III.1: Adaptive Law:

$$
\dot{\hat{A}}_{11}=-\Gamma \hat{e}_{1} x_{1}^{T}+\gamma_{0} \hat{B}_{0}^{T} \operatorname{Pex}_{1}^{T},
$$

where $\Gamma=\operatorname{diag}\left[\begin{array}{llll}\gamma_{1} & \gamma_{2} & \ldots & \gamma_{n_{1}}\end{array}\right], \gamma_{i}>0, i=1,2, \ldots, n_{1}$, and $\gamma_{0}>0$, results in $\lim _{t \rightarrow \infty} e(t)=0$.

Proof: The following tentative Lyapunov function is chosen:

$$
V=\frac{1}{2}\left(\hat{e}_{1}^{T} \Gamma \hat{e}_{1}+\gamma_{0} e^{T} P e+\operatorname{trace}\left(\Phi_{A}^{T} \Phi_{A}\right)\right) .
$$

It can be readily verified that if $A_{m}$ is stable, so is $\gamma_{0} A_{m}$, where $\gamma_{0}>0$. The Lyapunov Matrix Equation can now be modified as:

$$
\gamma_{0}\left(A_{m}^{T} P+P A_{m}\right)=-Q,
$$

or $A_{m}^{T} P+P A_{m}=-\bar{Q}$, where $\bar{Q}=Q / \gamma_{0}$.

Taking the first derivative of $V$ results in:

$$
\begin{aligned}
\dot{V}= & -\hat{e}_{1}^{T} \Gamma \Lambda \hat{e}_{1}+\Gamma \hat{e}_{1} \Phi_{A} x_{1}-e^{T} \bar{Q} e \\
& +\gamma_{0} e^{T} P \bar{B} \Phi_{A} x_{1}+\operatorname{trace}\left(\Phi_{A}^{T} \Phi_{A}\right) \\
= & -\hat{e}_{1}^{T} \Gamma \Lambda \hat{e}_{1}-e^{T} \bar{Q} e \\
& +\operatorname{trace}\left(\Phi_{A}^{T}\left(\Gamma \hat{e}_{1} x_{1}^{T}+\gamma_{0} \bar{B}^{T} P e x_{1}^{T}+\dot{\Phi}_{A}\right)\right) \\
\leq & -\lambda_{\Gamma \Lambda}\left\|\hat{e}_{1}\right\|^{2}-\lambda_{\bar{Q}}\|e\|^{2} \leq 0 .
\end{aligned}
$$

Hence all the signals are bounded. By integrating $V$ from 0 to $\infty$, it can be readily shown that $\left(\hat{e}_{1}, e\right) \in \mathcal{L}^{2}$. The theorem can now be proved using standard arguments.

\section{Comments:}

1 . The controller (13) is implemented by replacing the term $\dot{\hat{A}}_{11}$ with the expression (14) It can be readily verified that this controller has the same structure as the ideal certaintyequivalence controller (11), and only differs from the latter in terms that are bounded and depend on $\hat{e}_{1}(t)$ that has been demonstrated to tend to zero asymptotically.

2. It is important to note that the stability proof will go through even in the case when $\gamma_{0}$ is arbitrarily small. In practice this means that one can adjust the parameters using the observer only.

3. Case when $A_{11}=0$ and $A_{12}=I$ (controllable canonical form), and when $B_{2}=\bar{B}_{2} D$ where $\operatorname{rank}\left(\bar{B}_{2} D\right)=n_{2}$ and $A_{21}, A_{22}$ and $D$ are unknown, can be studied using the same approach as for the relative degree one plants. The major difference is the control law that would be of the form:

$$
\begin{aligned}
u= & \left(\bar{B}_{2} \hat{D}\right)^{-1}\left(\bar{B}_{2} \hat{D}\left(\bar{B}_{2} \hat{D}\right)^{T}\right)^{-1}\left(-\hat{A}_{21} x_{1}-\hat{A}_{22} x_{2}\right. \\
& \left.-K_{1} x_{1}-K_{2} x_{2}+K_{1} r\right),
\end{aligned}
$$

and the parameter adjustment laws would have been derived from the reduced-order observer:

$$
\dot{\hat{x}}_{2}=\Lambda_{2} \hat{e}_{2}+\hat{A}_{21} x_{1}+\hat{A}_{22} x_{2}+\bar{B}_{2} \hat{D} u,
$$

where $\Lambda_{2}=\Lambda_{2}^{T}<0$.

4. The proposed approach can be readily extended to the following case:

$$
\dot{x}=\left[\begin{array}{ccc}
A_{11} & A_{12} & 0 \\
A_{21} & A_{22} & A_{23} \\
A_{31} & A_{32} & A_{33}
\end{array}\right] x+\left[\begin{array}{c}
0 \\
B_{2} \\
B_{3} D
\end{array}\right] u,
$$

where $x_{i} \quad: \quad \mathbb{R}^{+} \quad \rightarrow \quad \mathbb{R}^{n_{i}}, \quad n_{1}=n_{2}$, $\operatorname{rank}\left(\left[\left(A_{12} B_{2}\right)^{T}\left(B_{3} D\right)^{T}\right]^{T}\right)=n_{1}+n_{3}$, and $A_{11}, A_{31}, A_{32}, A_{33}$ and $D$ are unknown.

\section{Extensions to Nonlinear System Dynamics}

In this section it will be shown that the extension of the reduced-order observer approach to three classes of nonlinear plants is straightforward. These classes include: (i) Relative degree one plants; (ii) Plants in controllable canonical form; and (iii) Relative degree two plants.

\section{A. Relative Degree One Plants}

In this case the plant dynamics is of the form:

$$
\begin{aligned}
& \dot{x}_{1}=f_{o}(x)+g_{o}(x) u \\
& \dot{x}_{2}=F(x) a+g(x) D u
\end{aligned}
$$

where $x_{i}: \mathbb{R}^{+} \rightarrow \mathbb{R}^{n_{i}}, i=1,2, n_{1}+n_{2}=n, u$ : $\mathbb{R}^{+} \rightarrow \mathbb{R}^{m}, f_{o}: \mathbb{R}^{n} \rightarrow \mathbb{R}^{n_{1}}, g_{o}: \mathbb{R}^{n} \rightarrow \mathbb{R}^{n_{1} \times m}$, $F: \mathbb{R}^{n} \rightarrow \mathbb{R}^{n_{2} \times p}$, and $g: \mathbb{R}^{n} \rightarrow \mathbb{R}^{n_{2} \times m}$. In the above equation, vector $a \in \mathbb{R}^{p}$ and matrix $D=\operatorname{diag}\left[\begin{array}{llll}d_{1} & d_{2} & \ldots & d_{n}\end{array}\right]$ are unknown.

The following assumption is made regarding the plant:

\section{Assumption 3:}

(a) State of the system is available at every instant;

(b) $f_{o}(x), g_{o}(x), F(x)$ and $g(x)$ are sufficiently smooth;

(c) $m \geq n$;

(d) $d \in \mathcal{S}_{d}$; and

(e) Let $\tilde{g}(x)=\left[g_{o}(x)^{T}(g(x) D)^{T}\right]^{T} \cdot \tilde{g}(x) \tilde{g}(x)^{T}$ is invertible for all $x$ on a domain and all $d \in \mathcal{S}_{d}$.

The reference model in this case is also of the form (2).

Now the following control objective is considered:

Control Objective: Design a control signal $u(t)$ such that all the signals in the system are bounded and the tracking error $e=x-x_{m}$ converges to zero asymptotically despite the uncertainty in $a$ and $D$.

Ideal Controller Design: In the case when $a$ and $D$ are known, the following controller achieves the objective:

$u=\tilde{g}(x)^{T}\left(\tilde{g}(x) \tilde{g}^{T}(x)\right)^{-1}\left(-\left[\begin{array}{c}f_{o}(x) \\ F(x) a\end{array}\right]+A_{m} x+B_{m} r\right)$. 
Parameter Estimation: In this case the observer is chosen in the form:

$$
\dot{\hat{x}}_{2}=F(x) \hat{a}+g(x) U \hat{d}+\Lambda_{2} \hat{e}_{2},
$$

where $\Lambda_{2}=\Lambda_{2}^{T}<0$ and $\hat{e}_{2}=\hat{x}_{2}-x_{2}$.

\section{Adaptive Laws:}

$$
\begin{aligned}
\dot{\hat{a}} & =-\Gamma_{1} F^{T}(x) \hat{e}_{2} \\
\dot{\hat{d}} & =\operatorname{Proj}_{\mathcal{S}_{d}}\left\{-\Gamma_{2} U g^{T}(x) \hat{e}_{2}\right\},
\end{aligned}
$$

where $\Gamma_{i}=\Gamma_{i}^{T}>0$, can be readily shown to result in boundedness of parameter estimates and $\hat{e}_{2} \in \mathcal{L}^{\infty} \cap \mathcal{L}^{2}$.

Coordinate Transformation: The following coordinate transformation is introduced:

$$
z_{1}=x_{1}, \quad z_{2}=\hat{x}_{2},
$$

resulting in

$$
\begin{aligned}
& \dot{z}_{1}=f_{o}(x)+g_{o}(x) u \\
& \dot{z}_{2}=F(x) \hat{a}+g(x) U \hat{d}+\Lambda_{2} \hat{e}_{2} .
\end{aligned}
$$

Adaptive Control: Let $\hat{\tilde{g}}(x)=\left[g_{o}(x)^{T}(g(x) \hat{D})^{T}\right]^{T}$. The control law is now chosen as:

$u=\tilde{\hat{g}}(x)^{T}\left(\tilde{\hat{g}}(x) \tilde{\hat{g}}^{T}(x)\right)^{-1}\left(-\left[\begin{array}{c}f_{o}(x) \\ F(x) \hat{a}\end{array}\right]+A_{m} x+B_{m} r\right)$.

resulting in:

$$
\dot{z}=A_{m} z+B_{m} r+\left[\begin{array}{c}
-A_{12}^{*} \hat{e}_{2} \\
\left(\Lambda_{2}-A_{22}^{*}\right) \hat{e}_{2}
\end{array}\right] .
$$

Upon choosing $e_{m i}=z_{i}-x_{m i}, i=1,2$, and subtracting the reference model, one obtains:

$$
\dot{e}_{m}=A_{m} e_{m}+\left[\begin{array}{c}
-A_{12}^{*} \\
\Lambda_{2}-A_{22}^{*}
\end{array}\right] \hat{e} .
$$

Using the BIBO arguments it follows that $e_{m}$ is bounded, and the proof that $\lim _{t \rightarrow \infty} e(t)=0$ is straightforward. The only difference with respect to the linear case is that $z$ rather than $x$ is used in the control law.

\section{B. Controllable Canonical Form}

This class of plants requires special attention since many of the practical systems can be transformed into this form.

Let the plant dynamics be of the form:

$$
\begin{aligned}
& \dot{x}_{1}=x_{2} \\
& \dot{x}_{2}=F(x) a+g(x) D u,
\end{aligned}
$$

where $x_{i}: \mathbb{R}^{+} \rightarrow \mathbb{R}^{n_{i}}, i=1,2, n_{1}=n_{2}, n_{1}+n_{2}=$ $n, u: \mathbb{R}^{+} \rightarrow \mathbb{R}^{m}, F: \mathbb{R}^{n} \rightarrow \mathbb{R}^{n_{2} \times p}$, and $g: \mathbb{R}^{n} \rightarrow$ $\mathbb{R}^{n_{2} \times m}$. In the above equation, vector $a \in \mathbb{R}^{p}$ and matrix $D=\operatorname{diag}\left[\begin{array}{llll}d_{1} & d_{2} & \ldots & d_{n}\end{array}\right]$ are unknown.

The following assumption is made regarding the plant:

\section{Assumption 4:}

(a) State of the system is available at every instant;

(b) $F(x)$ and $g(x)$ are sufficiently smooth; (c) $m \geq n$;

(d) $d \in \mathcal{S}_{d}$; and

(e) $(g(x) D)(g(x) D)^{T}$ is invertible for all $x$ on a domain and all $d \in \mathcal{S}_{d}$.

Let the reference model be of the form:

$$
\dot{x}_{m}=A_{m} x_{m}+B_{m} r,
$$

where $x: \mathbb{R}^{+} \rightarrow \mathbb{R}^{n}$,

$$
A_{m}=\left[\begin{array}{cc}
0 & I \\
-K_{1} & -K_{2}
\end{array}\right], B_{m}=\left[\begin{array}{c}
0 \\
K_{1}
\end{array}\right] .
$$

and $r: \mathbb{R}^{+} \rightarrow \mathbb{R}^{n_{2}}$, denotes a vector of bounded piece-wise continuous reference inputs.

Now the following control objective is considered:

Control Objective: Design a control signal $u(t)$ such that all the signals in the system are bounded and the tracking error $e=x-x_{m}$ converges to zero asymptotically despite the uncertainty in $a$ and $D$.

Ideal Controller Design: In the case when $a$ and $D$ are known, the following controller achieves the objective:

$$
\begin{aligned}
u= & (g(x) D)^{T}\left(g(x) D(g(x) D)^{T}\right)^{-1}\left(-F(x) a-K_{1} x_{1}\right. \\
& \left.-K_{2} x_{2}+K_{1} r\right)
\end{aligned}
$$

Parameter Estimation and Adaptive Laws: In this case the observer and adaptive laws for parameter adjustment are the same as in the case of relative degree one nonlinear plants.

Coordinate Transformation is the same as above

$$
z_{1}=x_{1}, \quad z_{2}=\hat{x}_{2}
$$

but in this case it results in:

$$
\begin{aligned}
& \dot{z}_{1}=z_{2}+\hat{e}_{2} \\
& \dot{z}_{2}=F(x) \hat{a}+g(x) U \hat{d}+\Lambda_{2} \hat{e}_{2} .
\end{aligned}
$$

Adaptive Control: The control law is now chosen as:

$$
\begin{aligned}
u= & (g(x) \hat{D})^{T}\left(g(x) \hat{D}(g(x) \hat{D})^{T}\right)^{-1}\left(-F(x) \hat{a}-K_{1} x_{1}\right. \\
& \left.-K_{2} x_{2}+K_{1} r\right)
\end{aligned}
$$

resulting in:

$$
\begin{aligned}
\dot{z}_{1} & =z_{2}+\hat{e}_{2} \\
\dot{z}_{2} & =\Lambda_{2} \hat{e}_{2}-K_{1} x_{1}-K_{2} x_{2}+K_{1} r \\
& =-K_{1} z_{1}-K_{2} z_{2}+K_{1} r+\left(\Lambda_{2}+K_{2}\right) \hat{e}_{2} .
\end{aligned}
$$

Upon choosing $e_{m i}=z_{i}-x_{m i}, i=1,2$, and subtracting the reference model, one obtains:

$$
\begin{aligned}
& \dot{e}_{m 1}=e_{m 2}+\hat{e}_{2} \\
& \dot{e}_{m 2}=-K_{1} e_{m 1}-K_{2} e_{m 2}+\left(\Lambda_{2}+K_{2}\right) \hat{e}_{2}
\end{aligned}
$$

Using the BIBO arguments it follows that $e_{m}$ is bounded, and the proof that $\lim _{t \rightarrow \infty} e(t)=0$ is straightforward. 


\section{Relative Degree Two Plants with Unmatched Uncertainty}

In this case the system model is of the form:

$$
\begin{aligned}
& \dot{x}_{1}=F\left(x_{1}\right) a+f_{o}\left(x_{2}\right) \\
& \dot{x}_{2}=f(x)+g(x) u
\end{aligned}
$$

where $x_{i}: \mathbb{R}^{+} \rightarrow \mathbb{R}^{n_{i}}, i=1,2, n_{1}=n_{2}, n_{1}+n_{2}=n$, $u: \mathbb{R}^{+} \rightarrow \mathbb{R}^{m}, F: \mathbb{R}^{n} \rightarrow \mathbb{R}^{n_{1} \times p}, f_{o}: \mathbb{R}^{n} \rightarrow \mathbb{R}^{n_{1}}$, $f: \mathbb{R}^{n} \rightarrow \mathbb{R}^{n_{2}}, g: \mathbb{R}^{n} \rightarrow \mathbb{R}^{n_{2} \times m}$. In the above equation, vector $a \in \mathbb{R}^{p}$ is unknown.

It is first noted that $F\left(x_{1}\right) a=\sum_{i=1}^{N} f_{i}\left(x_{1}\right) a_{i}$, and that

$$
J_{x_{1}}\left(x_{1}, a\right)=\frac{\partial F\left(x_{1}\right) a}{\partial x_{1}}=\sum_{i=1}^{N} a_{i} \frac{\partial f_{i}\left(x_{1}\right)}{\partial x_{1}}
$$

where $F\left(x_{1}\right)=\left[f_{1}\left(x_{1}\right) f_{2}\left(x_{1}\right) \ldots f_{N}\left(x_{1}\right)\right]$. We also have:

$$
J_{x_{2}}\left(x_{2}\right)=\frac{\partial f_{o}\left(x_{2}\right)}{\partial x_{2}} \text {. }
$$

Assumption 5: $\quad J_{x_{2}}\left(x_{2}\right) g(x)$ is invertible $\forall x$ on a domain. Control Objective: Similarly as in the linear case, the objective is to design a control signal $u(t)$ such that $x_{2}(t)$ is bounded and the state $x_{1}(t)$ of the plant follows asymptotically the state $x_{m 1}(t)$ of the reference model (9), (10).

The ideal controller is designed next. Let

$$
z_{1}=x_{1}, \quad z_{2}=F_{1}\left(x_{1}\right) a+f_{o}\left(x_{2}\right) .
$$

It follows that

$\dot{z}_{1}=z_{2}$

$\dot{z}_{2}=J_{x_{1}}\left(x_{1}, a\right)\left(F\left(x_{1}\right) a+f_{o}\left(x_{2}\right)\right)+J_{x_{2}}\left(x_{2}\right)(f(x)+g(x) u)$.

Ideal Controller: Ideal controller is now chosen in the form:

$$
u=\tilde{g}_{o}^{T}(x)\left(\tilde{g}_{o}(x) \tilde{g}_{o}^{T}(x)\right)^{-1}\left(-J_{x_{1}}\left(x_{1}, a\right)\left(F\left(x_{1}\right) a+f_{o}\left(x_{2}\right)\right)\right.
$$$$
\left.-J_{x_{2}}\left(x_{2}\right) f(x)-K_{1} z_{1}-K_{2} z_{2}+K_{1} r\right) \text {, }
$$

where $\tilde{g}_{o}(x)=J_{x_{2}}\left(x_{2}\right) g(x)$, and can be readily shown to achieve the control objective.

In this case the focus will be on minimal parameterization with a local observer.

Local Observer: The observer is chosen in the form:

$$
\dot{\hat{x}}_{1}=F\left(x_{1}\right) \hat{a}+f_{o}\left(x_{2}\right)-\Lambda \hat{e}_{1},
$$

where $\Lambda=\operatorname{diag}\left[\begin{array}{llll}\lambda_{1} & \lambda_{2} & \ldots & \lambda_{n_{1}}\end{array}\right]$ and $\lambda_{i}>0$.

Error Model: Upon subtracting (17) from the observer equation, and defining $\phi_{a}=\hat{a}-a$, one obtains:

$$
\dot{\hat{e}}_{1}=-\Lambda \hat{e}_{1}+F\left(x_{1}\right) \phi_{a} \text {. }
$$

The coordinate transformation is now chosen in the form:

$$
z_{1}=\hat{x}_{1}, \quad z_{2}=F\left(x_{1}\right) \hat{a}+f_{o}\left(x_{2}\right),
$$

resulting in:

$$
\begin{aligned}
\dot{z}_{1}= & z_{2} \\
\dot{z}_{2}= & F\left(x_{1}\right) \dot{\hat{a}}+J_{x_{1}}\left(x_{1}, \hat{a}\right)\left(F\left(x_{1}\right)\left(\hat{a}-\phi_{a}\right)\right. \\
& \left.\quad+f_{o}\left(x_{2}\right)\right)+J_{x_{2}}\left(x_{2}\right)(f(x)+g(x) u)
\end{aligned}
$$

The control law is now chosen in the form:

$$
\begin{aligned}
u= & \tilde{g}_{o}^{T}(x)\left(\tilde{g}_{o}(x) \tilde{g}_{o}^{T}(x)\right)^{-1}\left(-F\left(x_{1}\right) \dot{\hat{a}}\right. \\
& \left.-J_{x_{1}}\left(x_{1}, a\right)\left(F\left(x_{1}\right) \hat{a}+f_{o}\left(x_{2}\right)\right)-J_{x_{2}}\left(x_{2}\right) f(x)+v\right)
\end{aligned}
$$

Upon substituting this control law and subtracting the reference model, one obtains:

$$
\begin{aligned}
& \dot{e}_{1}=e_{2} \\
& \dot{e}_{2}=-K_{1} e_{1}-K_{2} e_{2}-\left(J_{x_{1}}\left(x_{1}, \hat{a}\right)+\Lambda\right) F\left(x_{1}\right) \phi_{a} .
\end{aligned}
$$

By choosing $\bar{B}=\left[\begin{array}{ll}0 & I\end{array}\right]^{T}$, this can be rewritten as:

$$
\dot{e}=A_{m} e-\bar{B}\left(J_{x_{1}}\left(x_{1}, \hat{a}\right)+\Lambda\right) F\left(x_{1}\right) \phi_{a} .
$$

Theorem IV.1: Adaptive Law:

$$
\dot{\hat{a}}=-\Gamma \hat{e}_{1} x_{1}^{T}+\gamma_{0}\left(J_{x_{1}}\left(x_{1}, \hat{a}\right)+\Lambda\right) \bar{B}^{T} \operatorname{Pex}_{1}^{T},
$$

where $\Gamma=\operatorname{diag}\left[\begin{array}{llll}\gamma_{1} & \gamma_{2} & \ldots & \gamma_{n_{1}}\end{array}\right], \gamma_{i}>0, i=1,2, \ldots, n_{1}$, and $\gamma_{0}>0$, results in $\lim _{t \rightarrow \infty} e(t)=0$.

This theorem can be proved along the same lines as theorem III.1.

\section{CONCLUSIONS}

In this paper a new systematic procedure is developed for the design of stable adaptive controllers using local reduced-order adaptive observers. It is shown that, for the class of plants considered in the paper, even when the unknown parameters are estimated using such lower-order observers, the resulting closed-loop system will be stable, and the asymptotic convergence of the tracking error to zero is guaranteed.

It is shown in the paper that the proposed approach is applicable to the plants whose multivariable relative degree is up to two. In the case of relative degree one plants, the proposed approach is based on a suitable coordinate transformation after which the analysis is carried out on a system that combines actual and estimated states. In the case of relative degree two plants, the approach is based on a coordinate transformation and a local observer, and is shown to result in parameter adjustment that depends on both the estimation and tracking errors. It is also shown that the adaptive gain for the tracking error adjustment can be made arbitrarily small, so that, in practice, the adjustment can be carried out using the estimation error only. This demonstrates that the certainty-equivalence principle carries over to this case as well.

Our future work includes extensions of the proposed approach to nonlinear plant models of higher relative degrees.

\section{REFERENCES}

[1] K. S. Narendra and A. M. Annaswamy, Stable Adaptive Systems, Prentice-Hall Inc., Englewood Cliffs, NJ 07632, 1989.

[2] S. Sastry and M. Bodson, Adaptive Control: Stability, Convergence and Robustness, Prentice-Hall, Englewood Cliffs, NJ 07632, 1989.

[3] M. Krstić, I. Kanellakopoulos, and P. Kokotović, Nonlinear and Adaptive Control Design, John Wiley \& Sons, 1995.

[4] A. Isidori, Nonlinear Control Systems, Springer-Verlag, 1989.

[5] J. D. Bošković, L. Chen and R. K. Mehra, "Adaptive Control Design for a Class of Non-Affine Models Arising in Flight Control", AIAA J. Guidance, Control \& Dynamics, 27(2), pp. 209-217, March 2004. 